\title{
PREPARATION AND CHARACTERIZATION OF TRIFLUOPERAZINE LOADED TRANSDERMAL PATCHES FOR SUSTAINED RELEASE
}

\author{
ANJU PARAMBIL ${ }^{1}$, SEENIVASAN PALANICHAMY ${ }^{*}$, ARUL KUTTALINGAM ${ }^{3}$, VELLAPANDIAN CHITRA ${ }^{4}$
}

${ }^{1}$ College of Pharmaceutical Sciences, Government Medical College, Kozhikode 673008, Kerala, ${ }^{2}$ Sri Ramachandra Faculty of Pharmacy, Sri Ramachandra Institute of Higher Education and Research, Porur, Chennai 600116, Tamil Nadu, India, ${ }^{3}$ College of Pharmaceutical Sciences, Government Medical College, Kottayam 686008, Kerala, ${ }^{4}$ SRM College of Pharmacy, SRM University, Kattankulathur 603203, Tamilnadu *Email: seenumelur@gmail.com

Received: 13 Jun 2021, Revised and Accepted: 19 Aug 2021

\section{ABSTRACT}

Objective: The purpose of the present study was to formulate and evaluate the polymeric transdermal delivery system of antipsychotic drug Trifluoperazine (TFP) for sustained drug release.

Methods: A transdermal patch loaded with (TFP) was formulated by solvent casting technique. Polyvinyl pyrrolidone (PVP) K-30 and ethyl cellulose (EC) was used as a polymeric matrix with different ratios. Di n-butyl phthalate was used as a plasticizer. The parameters such as thickness, folding endurance and weight variation of the prepared patches were studied. The interaction study by attenuated total reflectance-infrared (ATRIR) spectroscopy, X-ray diffraction and thermal analysis by differential scanning calorimetry (DSC) were performed. In vitro drug release study was performed by modified paddle over-disc technique.

Results: The infrared spectroscopic study confirmed the absence of any chemical interaction between TFP and selected polymers. All the prepared formulations showed folding endurance values ranging from 130-162 and a satisfactory drug loading of $90-95 \%$. In in vitro drug release study, formulations PE-3 and PE-4 exhibited a sustained and stable cumulative release of $54 \%$ and $48 \%$ respectively, at the end of 24 h. The DSC and XRD analysis proved the partial conversion of the drug from crystalline to amorphous form when integrated into the polymeric matrix.

Conclusion: The prepared transdermal formulations using polymers PVP and ethyl cellulose demonstrated their ability to sustain the release of TFP. The developed formulation could be exploited for multiday therapy of TFP for the effective treatment of schizophrenia with a simplified dosing regimen and enhanced patient compliance.

Keywords: Trifluoperazine, Transdermal patches, Psychiatric disorders, Solvent casting, Sustained release

(C) 2021 The Authors. Published by Innovare Academic Sciences Pvt Ltd. This is an open access article under the CC BY license (https://creativecommons.org/licenses/by/4.0/) DOI: https://dx.doi.org/10.22159/ijap.2021v13i6.42413. Journal homepage: https://innovareacademics.in/journals/index.php/ijap

\section{INTRODUCTION}

Trifluoperazine (TFP) is an antipsychotic drug; it is widely used to treat psychotic conditions of acute and chronic schizophrenia, since 1950s. TFP is the first generation (typical antipsychotics) medication that has proved to be a potent phenothiazine derivative with a strong affinity to D2 receptors $[1,2]$. New generation antipsychotic drugs are available for claiming fewer extrapyramidal side effects. TFP still remains to be an inexpensive and widely used drug in the management of schizophrenia and anxiety. Even though TFP is well absorbed orally, it is reported to undergo extensive firstpass metabolism in the liver. Psychiatric disorders often demand long term drug treatment, and compliance with the prescribed medication is a severe issue for mental illness people. Nonadherence to prescribed medication in psychiatric patients is mainly due to factors like undesirable side effects, dosing regimen, route of administration, nature of illness, belief systems and personal attributes [3]. The transdermal delivery of psychotropics is an under-investigated area of research. The approach of developing antipsychotics in the form of a sustained release transdermal patch is promising as the occurrence of non compliance with medication and relapse is high in patients with mental illness [3].

In comparison with both the parenteral and oral routes, transdermal delivery seems to have several benefits. Transdermal systems offer advantages like controlled drug release, improved bioavailability, minimal side effects, reduced dosing frequency and evasion of first pass metabolism [4-6]. Moreover, non-invasive transdermal devices are self-administered and the drug action can be rapidly terminated by removing the system from the skin. Transdermal drug delivery also provides a long-standing and steady systemic absorption pattern, minimising several side effects caused by the peak valley profile of plasma drug concentrations [7-10] and the fabrication techniques are usually simple and of less cost.
The drug delivery technology in the form of a transdermal patch has developed immensely since 1979 when the FDA approved the first transdermal patch of scopolamine for motion sickness. Some significant highlights in the progress of transdermal delivery include nitroglycerin for angina, nicotine for smoking cessation, fentanyl as analgesic and lidocaine [11]. The major classes of transdermal patches include drug in different types such as adhesive, reservoir, matrix, micro reservoir and membrane-matrix hybrid [12, 13]. Among all these, matrix type transdermal patches are well accepted, due to ease of manufacturing [13] and they also minimise the risk of dose dumping in case of any failure in the system.

The present investigation focused on developing a sustained-release transdermal system of TFP that can deliver the drug over multiple days to simplify the dose regimen and improve patient compliance. In this study, a combination of hydrophobic polymer ethyl cellulose (EC) was used along with a soluble polymer polyvinyl pyrrolidone (PVP) to sustain the release of the drug from the device. EC is one of the extensively used coating materials to achieve controlled release of drug from solid dosage forms [14]. Ethylcellulose is a water insoluble polymer with excellent safety records and global compendial acceptance [15]. PVP is a non-ionic polymer exhibiting varying solubility range and high swelling properties and blending of PVP with a number of other polymers are reported to achieve flexibility, improve film-forming ability and/or drug controlled release of drug from the delivery device [16, 17]. Combining PVP with a retarding polymer like EC is reported to control the release of drug from the matrix and it also retard the crystallisation of drug [18, 19]. For the fabrication of films, solvent casting method employing organic solvents included with long chain esters as plasticizers are usually used [14]. Biodegradable plasticizers like dibutyl phthalate (DBP) are reported to enhance the thermal and mechanical properties when incorporated in a polymeric film $[14,20]$. In this study matrix patches of TFP with varying ratios of PVP: EC combinations are fabricated using DBP as the plasticizer. The prepared formulations were 
compared by different in vitro evaluations to choose the best combination for sustained transdermal delivery of TFP.

\section{MATERIALS AND METHODS}

\section{Materials}

Trifluoperazine dihydrochloride was purchased from Tokyo Chemical Industry, Japan. Ethyl cellulose was purchased from the Dow Chemical Company, USA, which has a viscosity of $100 \mathrm{cP}$. Polyvinyl pyrrolidone (PVP K-30) was obtained from Loba Chemie, Mumbai. Chloroform and Di n-butyl phthalate were obtained from the Merck life sciences Pvt. Ltd, (Bengaluru) and Central drug house (P) Ltd, (New Delhi). The chemicals were employed as obtained without any other purification. All remaining reagents employed in this analysis were of analytical grade.

\section{Methods}

\section{Preparation of TFP loaded transdermal patch}

TFP loaded transdermal patch comprising varying proportions of PVP K-30 and EC was fabricated by the solvent casting technique. Preliminary studies were done with blank patches to select proper ratios of polymer and concentration of plasticizer to be incorporated. The prerequisite ratios of polymers were weighed and allowed to swell for $4 \mathrm{~h}$ in chloroform-methanol (9:1) solvent mixture. The total weight of the polymer in one formulation was kept $400 \mathrm{mg}$. Plasticizer Di n-butyl phthalate was incorporated at $30 \% \mathrm{w} / \mathrm{w}$ of polymer dry weight. The weighed amount of TFP was incorporated with the polymer blend, and this was stirred using a magnetic stirrer at $300 \mathrm{rpm}$ for $20 \mathrm{~min}$ to get a homogenous mixture. The mixture was ultrasonicated to remove air bubbles and slowly poured into glass moulds $(\mathrm{dm}-3 \mathrm{~cm})$ formerly dried with $4 \%$ $\mathrm{w} / \mathrm{v}$ PVA solution as backing [21]. A funnel was positioned over the glass mould in an inverted position to regulate the rate of evaporation and was dried at room temperature for $24 \mathrm{~h}$. The matrix films were detached from the mould and folded in aluminium foil and stored in a desiccator until used for further evaluation.

\section{Drug-excipient compatibility study}

The interaction of the drug with selected excipients was studied by Infrared spectroscopy. The IR spectra of pure drug TFP, individual polymers (EC and PVP K30) and physical mixture of drug with polymers were recorded by ATR-FT-IR (Jasco FT/IR-4700) over the spectral range of $4000-500 \mathrm{~cm}^{-1}[22,23]$. The FT-IR spectra of the formulated patches were also recorded and compared with that of pure drug.

\section{Evaluation of the physicochemical properties of the patches}

\section{Weight and thickness}

Six patches $\left(3 \mathrm{~cm}^{2}\right)$ from each batch were randomly chosen for the analysis of weight variation. Weights of the formulations were taken independently, and further, the average weight was measured. The thickness of the patches was evaluated at 3 different points utilizing a digital micrometer and the mean values were tabulated [24, 25].

\section{Folding endurance}

The mechanical strength of the patches can be evaluated by determining folding endurance. The analysis of folding endurance was carried out for all patches, and it was calculated manually. A strip of the patch was folded manually at the same position repeatedly until it was broken. The number of folding needed to break the film was tabulated as folding endurance [26].

\section{Drug content analysis}

A patch of $3 \mathrm{~cm}^{2}$ area was randomly taken and dissolved in $5 \mathrm{ml}$ of casting solvent, and the volume was diluted suitably and filtered by using Whatman filter paper. The drug quantity in each solution was then evaluated spectrophotometrically utilizing UV-Visible spectrophotometer (Shimadzu UV-1800, Japan) at $257 \mathrm{~nm}$. A control was also performed using drug-free films [14]. An average of three readings was tabulated.

\section{In vitro drug release study}

The dissolution studies of the formulated patches were carried out in the USP type 2 dissolution apparatus. The patches $\left(3 \mathrm{~cm}^{2}\right.$ area $)$ were fixed on a small glass plate at the bottom of the vessel. The $\mathrm{pH}$ 7.4 phosphate buffer medium $900 \mathrm{ml}$ was used and the temperature was maintained at $37 \pm 0.5{ }^{\circ} \mathrm{C}$ with paddle rpm of 75 . Samples were withdrawn at different time intervals and analysed utilizing a UVvisible spectrophotometer at $257 \mathrm{~nm}$ against a blank. Cumulative amounts of TFP released were plotted against time for all the formulations. The drug release pattern from different formulations was compared

\section{Thermal analysis with differential scanning colorimetry (DSC)}

Thermal analysis of TFP, physical mixture (TFP, EC and PVP K30) and the developed patch PE-3 and PE-4 was performed using differential scanning calorimetry (DSC) (Q 20, TA instrument). Specimens were heated from room temperature to $300{ }^{\circ} \mathrm{C}$ at a rate of $10^{\circ} \mathrm{C}$ per min in a nitrogen purge of $50 \mathrm{ml}$ per min [19].

\section{X-ray diffraction study (XRD)}

The X-Ray diffraction (XRD) study was mainly utilized to analyze the crystalline and stable dispersive behaviour of the drug TFP when dispersed in the patch. The X-ray diffractogram of TFP and the formulations PE-3 was recorded (Bruker model D8 advance) at 25 ${ }^{\circ} \mathrm{C}$ utilizing monochromatic copper $\mathrm{K}$-alpha radiation at $40 \mathrm{~mA}$ and $40 \mathrm{kV}$.

\section{Scanning electron microscopy (SEM) analysis}

Scanning electron microscopy analysis was employed for identifying the surface characteristics of the developed formulations PE-3 and PE-4. The specimens were analysed using SEM (JEOL JSM 6390) with a high vacuum and a high voltage of $15 \mathrm{kV}$.

\section{RESULTS AND DISCUSSION}

\section{Preparation of TFP loaded transdermal patch}

The formulations were prepared by solvent casting method varying the ratio of PVP: EC. From the results of preliminary studies carried out, the total content of polymer in each patch was maintained as $400 \mathrm{mg}$ and the plasticizer (Di n-butyl phthalate) concentration was kept constant as $30 \%$ of the polymer weight. As the objective of the study is to develop a patch releasing the drug gradually over multiple days, the concentration of the retarding polymer EC was gradually increased in the formulations as shown in table 1.

Table 1: Composition for the prepared trifluoperazine loaded transdermal patch

\begin{tabular}{lllll}
\hline Ingredients (PVP: EC ratio) & PE-1 (3:2) & PE-2 (2:3) & PE-3 (1:3) & PE-4 (1:5) \\
\hline PVP K-30 (mg) & 240 & 160 & 100 & 67 \\
Ethylcellulose (mg)? & 160 & 240 & 300 & 333 \\
Di n-butyl phthalate (ml)? & 0.12 & 0.12 & 0.12 & 350 \\
Trifluoperazine dihydrochloride (mg) & 20 & 20 & 20 & 0.12 \\
Chloroform: methanol (9:1) (ml) & 5 & 5 & 5 & 20 \\
\hline
\end{tabular}

\section{Drug-excipient compatibility study}

Interactions of drug with excipients play an important role in controlling the release of drugs from the formulation. ATR-FTIR technique has been used here to study the physical and chemical interactions between the drug and polymers used. The infrared spectra for the TFP, excipients (EC, PVP K30) and physical mixture of TFP, EC and PVP K-30 was recorded. The IR spectra of prepared 
formulations PE-3 and PE-4 was also analysed (fig. 1). The IR spectra of TFP exhibited bands of $\mathrm{CF}_{3}$ symmetric stretching at 1108,1143 and $1080 \mathrm{~cm}^{-1}$; C-S stretching at 678 and $754 \mathrm{~cm}^{-1}$; C-N stretching at 1250 $\mathrm{cm}^{-1}$; $\mathrm{C}_{-} \mathrm{CF}_{3}$ stretching at $1316 \mathrm{~cm}^{-1}$ and aromatic $\mathrm{C}=\mathrm{C}$ stretching at 1600,1565 and $1470 \mathrm{~cm}^{-1}[23,27]$. The spectrum of ethylcellulose shows characteristic absorption bands for-C-O-C-stretching vibration at $1052 \mathrm{~cm}^{-1}$ and $\mathrm{C}-\mathrm{H}$ stretching bands at $2871 \mathrm{~cm}^{-1}$ and $2969 \mathrm{~cm}^{-1}$. The absorption at $1374 \mathrm{~cm}^{-1}$ corresponds to $\mathrm{C}-\mathrm{H}$ bending. The infrared spectrum of PVP is characterized by a strong $\mathrm{C}=\mathrm{O}$ absorption peak from the amide group of PVP at $1650 \mathrm{~cm}^{-1}, \mathrm{C}-\mathrm{N}$ group appeared at $1287 \mathrm{~cm}^{-1}, \mathrm{C}-\mathrm{H}$ stretching and bending vibrations were observed at 2800-3000 and 1420-1460 $\mathrm{cm}^{-1}$, respectively.
From the FT-IR spectral analysis, it is evident that the characteristic peaks of the drug were intact in the physical mixture and the formulations (PE-3and PE-4) with only minor variations. The nonappearance of additional peaks in the formulations indicates the absence of any chemical incompatibilities between the drug and the polymers selected for the formulation development. However, the FTIR spectra for the formulations show overlapping of TFP and excipients bands and there are some very minor changes in the peaks in the range of $1000-1300 \mathrm{~cm}^{-1}$ probably due to some weak physical interactions between polymers and the drug, but this type of interaction did not interfere with the release of drug from the PVP: EC polymer matrix.

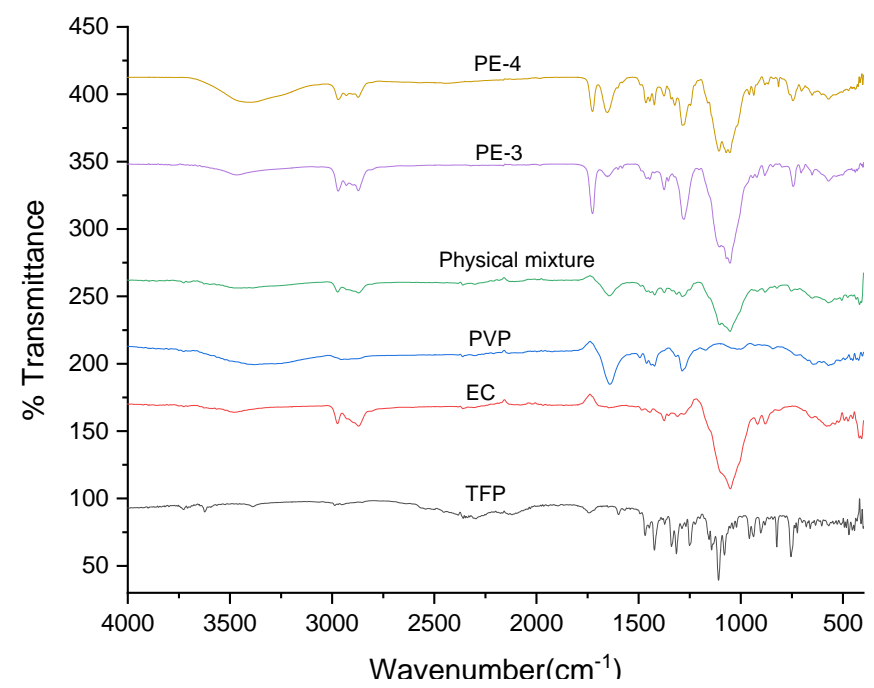

Fig. 1: FT-IR spectra of TFP; EC; PVP K-30; the physical mixture of (TFP, EC and PVP K-30); formulation PE-3 and PE-4

\section{Physicochemical characteristics of the transdermal patch}

The physicochemical characteristics of all the formulations were analysed. The thickness of the formulations was found to be in the range of 0.529 to $0.568 \mathrm{~mm}$. Moreover, the weight disparity was identified between 0.5098 to $0.5414 \mathrm{~g}$ (table 2). From the analysis, it was clear that all the PE formulations were more or less equivalent in weight and thickness.

Table 2: Results of physicochemical parameters of formulation PE-1 to PE-5

\begin{tabular}{lllll}
\hline Formulation code & Thickness in $(\mathbf{m m}) \mathbf{( n = 3 )}$ & Average weight $\mathbf{( g )}(\mathbf{n}=\mathbf{6})$ & Folding endurance $(\mathrm{n}=3)$ & Average drug content $(\mathbf{\%}) \mathbf{( n = 3 )}$ \\
\hline PE-1 & $0.537 \pm 0.002$ & $0.5414 \pm 0.0010$ & $130 \pm 3$ & $93.82 \pm 0.870$ \\
PE-2 & $0.546 \pm 0.004$ & $0.5296 \pm 0.0062$ & $142 \pm 2$ & $90.97 \pm 0.729$ \\
PE-3 & $0.568 \pm 0.005$ & $0.5226 \pm 0.0117$ & $156 \pm 4$ & $95.22 \pm 0.478$ \\
PE-4 & $0.529 \pm 0.005$ & $0.5098 \pm 0.0060$ & $152 \pm 2$ & $93.23 \pm 1.425$ \\
PE-5 & $0.531 \pm 0.003$ & $0.5193 \pm 0.0093$ & $162 \pm 3$ & $92.27 \pm 1.243$ \\
\hline
\end{tabular}

Data represent mean \pm SD

The folding endurance properties of all five patches were analysed, and their corresponding results were enlisted in table 2 . The folding endurance of all the five patches (PE-1 to PE-5), were found to be in the range of 130 to 162 indicating satisfactory flexibility and mechanical strength of the patch. Drug content was identified to be ranging from 90 to $95 \%$, showing that the drug was satisfactorily loaded and evenly distributed in the polymer matrix.

\section{In vitro drug release analysis}

The in vitro dissolution analysis was performed for all five patches and the results are plotted as percentage cumulative drug released (\% CDR) vs. time (fig. 2). Formulation PE-1 with PVP: EC ratio 3: 2 released 89\% TFP within $8 \mathrm{~h}$, due to high concentration of PVP K-30. PVP K-30 being a water-soluble polymer dissolves in the dissolution medium easily making the polymeric matrix porous leading to leach out of the drug from the matrix. As the concentration of hydrophobic polymer EC gradually increased, the release rate of TFP from the system was found to be retarding. Formulations PE-3 and PE-4 with PVP-EC ratio1:3 and 1:5 respectively resulted in a sustained and stable release of TFP from the polymeric matrix over a period of $24 \mathrm{~h}$ and was selected for further evaluations. These formulations could be exploited for multiday therapy of TFP which can simplify the dosage regimen and improve patient compliance in psychiatric patients. The matrix patch PE-5 with a PVP: EC ratio of 1:7 was showing a stagnant release of drug from the device after $8 \mathrm{~h}$ (only up to $20 \%$ ), owing to a high concentration of hydrophobic polymer that retard the drug release from the device once the surface drug was released.

\section{DSC analysis}

The DSC analysis is an important method to identify the thermal characteristics of the drug when incorporated in a formulation. Fig. 3 shows the DSC thermogram of pure TFP and matrix type transdermal patches (PE-3 and PE-4) containing TFP. From the 
obtained thermogram of neat TFP, it is evident that melting of TFP appears to start at approximately $240{ }^{\circ} \mathrm{C}$ followed by decomposition at about $250{ }^{\circ} \mathrm{C}[28,29]$. This observation is in concordance with the previous reports. In the thermogram of the formulations, the endothermic peak of TFP was found to be smaller as compared to pure drug; probably due to the minimal amount of TFP incorporated in the formulation. Moreover, the peaks have shown a small shift and broadening. This could be expected as the drug was dissolved completely in the polymeric solution during formulation development, leading to the formation of a molecular dispersion of the drug in the polymer mixture. However, the presence of endothermic peak of TFP in the formulation suggests that the crystallinity of the drug was not completely lost during the development of the formulations.

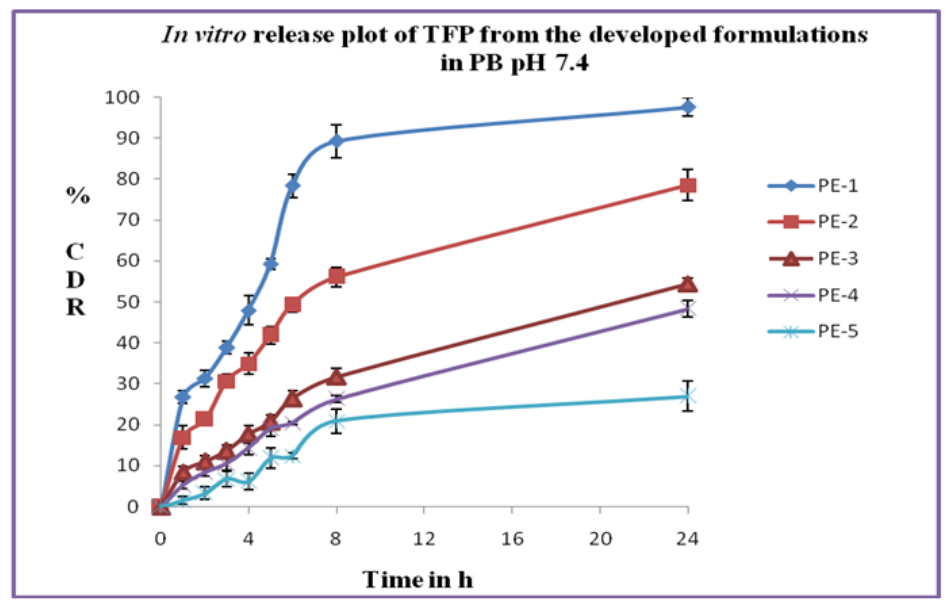

Fig. 2: In vitro drug release data of formulation $\mathrm{PE}-1$ to $\mathrm{PE}-5$, data represents mean $\pm \mathrm{SD}(\mathrm{n}=3$ )

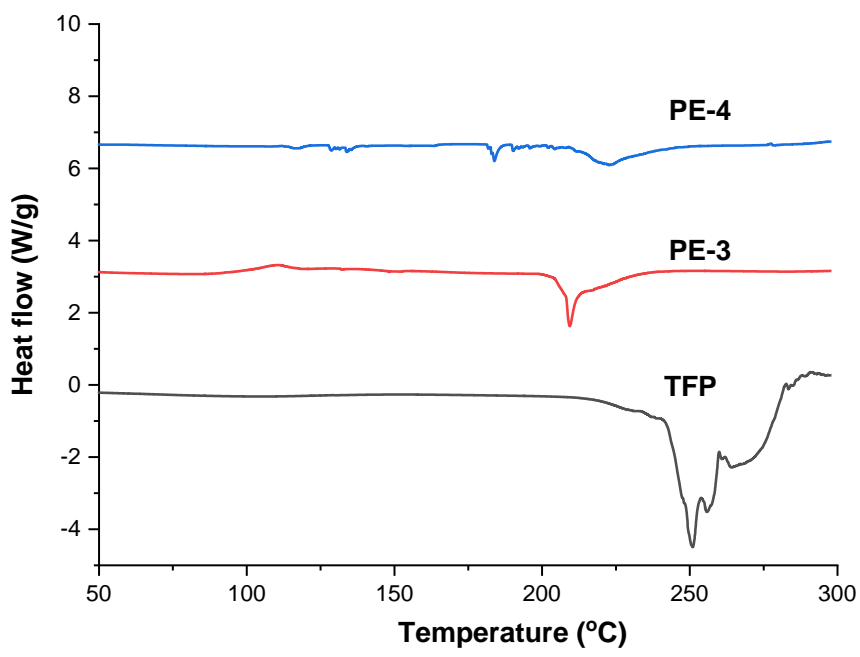

Fig. 3: DSC thermogram of TFP, formulation PE-3 and PE-4

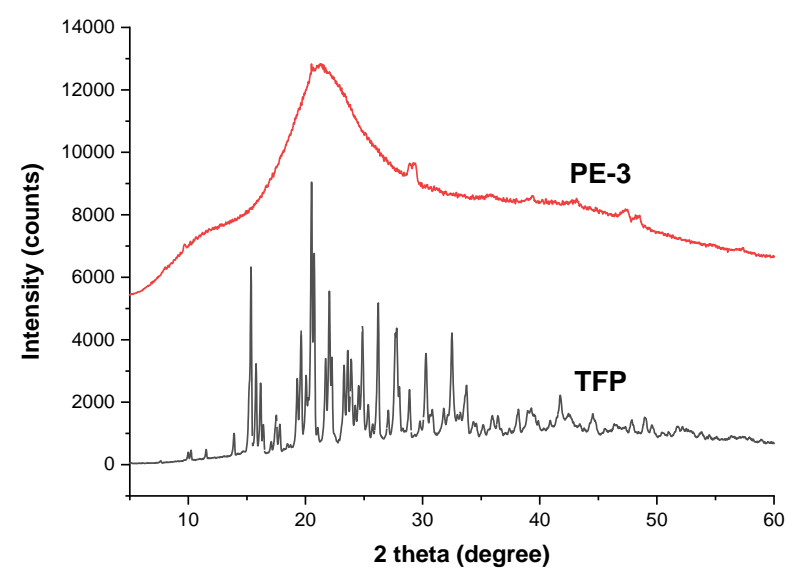

Fig. 4: XRD patterns of TFP and formulation PE-3 


\section{XRD analysis}

The crystallinity of neat TFP and TFP, when distributed in the formulation PE-3 was evaluated by XRD analysis and the recorded patterns are presented in fig. 4. The pure drug TFP shows the presence of sharp intense peaks in the diffraction pattern and the characteristic peaks of the crystal form of neat TFP was observed at $15.4^{\circ}, 20.5^{\circ}, 20.7^{\circ}, 22^{\circ}$ and $26.2^{\circ}(2 \theta)$. In the formulation, even though two peaks $\left(20.5^{\circ}, 20.7^{\circ}\right)$ were visible other characteristic peaks of TFP were found to be absent. Such a decrease in amplitude and broadening of the peaks are characteristic of the presence of amorphous substances. The earlier reports suggest that the presence of PVP K-30 can inhibit the crystallinity of the drug in the polymeric matrix [19]. Since DSC reports also confirm this observation, it can be assumed that partial conversion of TFP to amorphous form has taken place during formulation development.

\section{SEM analysis}

The SEM analysis of formulations PE-3 and PE-4 depicted a smooth surface with the absence of drug crystals or pores, indicating homogenous dispersion of drug in the patch. The Nonappearance of drug crystals on the surface also supports the results of DSC and XRD analysis suggesting molecular dispersion of drug in the polymeric matrix.

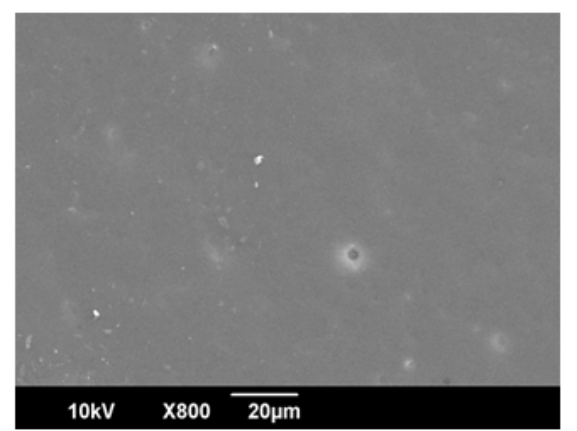

PE-3

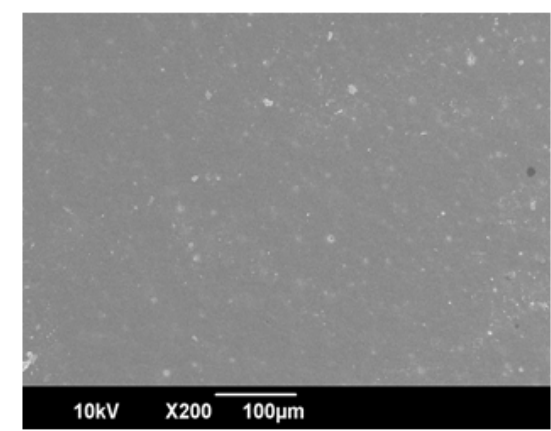

PE-4

Fig. 5: SEM image of formulation PE-3 and PE-4

\section{CONCLUSION}

The results of the present investigation suggest that a polymeric matrix with PVP: ethyl cellulose in the ratio $1: 3$ or above can effectively retard the release of TFP. As the concentration of EC in the polymeric matrix was enhanced, the release of TFP was identified as retarding. Different characterizations done on the developed formulations confirmed satisfactory drug loading and mechanical properties. The thermal analysis results suggest a change in crystallinity of the incorporated drug in the formulations. Formulations PE-3 and PE-4 with PVP-EC proportions of 1:3 and 1:5, respectively, can be subjected to further in vitro and in vivo evaluations to explore the potential of the developed system for effective transdermal delivery of TFP for multiple days and thereby increasing the patient compliance and bioavailability.

\section{FUNDING}

Nil

\section{AUTHORS CONTRIBUTIONS}

All the authors have contributed equally.

\section{CONFLICT OF INTERESTS}

The authors declare no conflict of interest.

\section{REFERENCES}

1. Koch K, Mansi K, Haynes E, Adams CE, Sampson S, Furtado VA. Trifluoperazine versus placebo for schizophrenia. Cochrane Database Syst Rev. 2014;(1):CD010226. doi: 10.1002/14651858.CD010226.pub2, PMID 24414883.

2. Joint formulary committee. British National Formulary. BMJ 2008;56:191-5.

3. Isaac M, Holvey C. Transdermal patches: the emerging mode of drug delivery system in psychiatry. Ther Adv Psychopharmacol. 2012;2(6):255-63. doi: 10.1177/2045125312458311, PMID 23983984.

4. Siafaka PI, Barmpalexis P, Lazaridou M, Papageorgiou GZ, Koutris E, Karavas E, et al. Controlled release formulations of risperidone antipsychotic drug in novel aliphatic polyester carriers: data analysis and modelling. Eur J Pharm Biopharm.
2015;94:473-84. doi: 10.1016/j.ejpb.2015.06.027, PMID 26159838. ejpb.2015.06.027.

5. Mazzitelli S, Pagano C, Giusepponi D, Nastruzzi C, Perioli L. Hydrogel blends with adjustable properties as patches for transdermal delivery. Int J Pharm. 2013;454(1):47-57. doi: 10.1016/j.ijpharm.2013.06.081, PMID 23856160.

6. Jalhan S, Kaur K, Kaur P, K Jain U. Formulation and in vitro evaluation of transdermal matrix patches of doxophylline. Asian J Pharm Clin Res. 2016;9(5):140-5. doi: 10.22159/ajpcr.2016.v9i5.12774.

7. Citrome L, Zeni CM, Correll CU. Patches: established and emerging transdermal treatments in psychiatry. J Clin Psychiatry. 2019;80(4). doi: 10.4088/JCP.18nr12554, PMID 31318185.

8. Jung E, Lee EY, Choi HK, Ban SJ, Choi SH, Kim JS, Yoon IS, Kim DD. Development of drug-in-adhesive patch formulations for transdermal delivery of fluoxetine: in vitro and in vivo evaluations. Int J Pharm. 2015;487(1-2):49-55. doi: 10.1016/j.ijpharm.2015.04.012, PMID 25863117.

9. Hardainiyan SW, Kumar KR, Nandy BC, Saxena RI. Design, Formulation and in vitro drug release from transdermal patches containing imipramine hydrochloride as model drug. Int J Pharm Pharm Sci. 2017;9(6):220-5. doi: 10.22159/ijpps.2017v9i6.16851.

10. Das PS, Saha P. Design and characterisation of transdermal patches of phenformin hydrochloride. Int J Curr Pharm Sci. 2017;9(6):90-3. doi: 10.22159/ijcpr.2017v9i6.23437.

11. Walter JR, Xu S. Therapeutic transdermal drug innovation from 2000 to 2014: current status and outlook. Drug Discovery Today. 2015;20(11):1293-9. 10.1016/j.drudis.2015.06.007, PMID 26116094.

12. Sachdeva V, Bai Y, Kydonieus A, Banga AK. Formulation and optimization of desogestrel transdermal contraceptive patch using crystallization studies. Int J Pharm. 2013;441(1-2):9-18. doi: $\quad 10.1016 / j . i j p h a r m .2012 .12 .014, \quad$ PMID 23262424. ijpharm.2012.12.014.

13. Mukherjee B, Mahapatra S, Gupta R, Patra B, Tiwari A, Arora P. A comparison between povidone-ethylcellulose and povidoneeudragit transdermal dexamethasone matrix patches based on in vitro skin permeation. Eur J Pharm Biopharm. 2005;59(3):47583. doi: 10.1016/j.ejpb.2004.09.009, PMID 15760728. 
14. Rajabalaya R, Rani S, David N, Khanam J, Nanda A. Effect of plasticizers on in vitro release and ex vivo permeation of chlorpheniramine maleate from ethyl cellulose polyvinyl pyrrolidone based matrix patches. Farmacia. 2013;61:975-90.

15. Koradia H, Butani S, Gohel M. Studies in oxcarbazepine microspheres employing plackett and burman design. Int J Pharm Pharm Sci. 2014;6:305-10.

16. Karki S, Kim H, Na SJ, Shin D, Jo K, Lee J. Thin films as an emerging platform for drug delivery. Asian J Pharm Sci. 2016;11(5):559-74. doi: 10.1016/j.ajps.2016.05.004

17. Jaipakdee N, Pongjanyakul TH, Limpongsa EK. Preparation and characterization of poly (vinyl alcohol)-poly (vinyl pyrrolidone) mucoadhesive buccal patches for delivery of lidocaine HCL. Int J App Pharm. 2018;10(1):115-23. doi: 10.22159/ijap.2018v10i1.23208.

18. Kandavilli S, Nair V, Panchagnula R. Polymers in transdermal drug delivery systems. Pharm Technol. 2002;26:62-81.

19. Suksaeree J, Siripornpinyo P, Chaiprasit S. Formulation, characterization, and in vitro evaluation of transdermal patches for inhibiting crystallization of mefenamic acid. J Drug Delivery. 2017;2017:7358042. doi: 10.1155/2017/7358042, PMID 29259828.

20. Vieira MGA, Da Silva MA, Dos Santos LO, Beppu MM. Naturalbased plasticizers and biopolymer films: a review. Eur Polym J. 2011;47(3):254-63. doi: 10.1016/j.eurpolymj.2010.12.011.

21. Arora P, Mukherjee B. Design, development, physicochemical, and in vitro and in vivo evaluation of transdermal patches containing diclofenac diethylammonium salt. J Pharm Sci. 2002;91(9):2076-89. doi: 10.1002/jps.10200, PMID 12210054.

22. Veras KS, Fachel FNS, Pittol V, Garcia KR, Bassani VL, dos Santos V, et al. Compatibility study of rosmarinic acid with excipients used in pharmaceutical solid dosage forms using thermal and non-thermal techniques. Saudi Pharm J. 2019;27(8):1138-45. doi: 10.1016/j.jsps.2019.09.010, PMID 31885473. jsps.2019.09.010.

23. Moffat AC, Osselton MD, Widdop B, Watts J. Clarke's analysis of drugs and poisons. Vol. II. London: Pharmaceutical Press; 2011. p. 2193-4.

24. Nair RS, Ling TN, Abdul Shukkoor MS, Manickam B. Matrix type transdermal patches of captopril: Ex vivo permeation studies through excised rat skin. J Pharm Res. 2013;6(7):774-9. doi: 10.1016/j.jopr.2013.07.003.

25. Bhatt DC, Dhake AS, Khar RK, Mishra DN. Development and in vitro evaluation of transdermal matrix films of metoprolol tartrate. Yakugaku Zasshi. 2008;128(9):1325-31. doi: 10.1248/yakushi.128.1325, PMID 18758147.

26. Parhi R, Suresh P. Transdermal delivery of diltiazem $\mathrm{HCl}$ from matrix film: effect of penetration enhancers and study of antihypertensive activity in rabbit model. J Adv Res. 2016;7(3):539-50. doi: 10.1016/j.jare.2015.09.001. PMID 27222758.

27. Rajesh P, Gunasekaran S, Gnanasambandan T, Seshadri S. Molecular structure and vibrational analysis of trifluoperazine by FT-IR, FT-Raman and UV-vis spectroscopies combined with DFT calculations. Spectrochim Acta A Mol Biomol Spectrosc. 2015;137:1184-93. doi: 10.1016/j.saa.2014.08.100, PMID 25305610.

28. British Pharmacopoeia. Commission. Vol. II. British Pharmacopoeia. London; 2016.

29. Post A, Warren RJ, Zarembo JE. Trifluoperazine hydrochloride. Anal Profiles Drug Substances. 1981;9:543-81. doi: 10.1016/S0099-5428(08)60153-7. 